\title{
Facial expression recognition and emotion understanding in children after neonatal open-heart surgery for transposition of the great arteries
}

\author{
JOHANNA CALDERON ${ }^{1}$ | NATHALIE ANGEARD ${ }^{1}$ | CHARLOTTE PINABIAUX ${ }^{1}$ | DAMIEN BONNET ${ }^{2}$ | ISABELLE \\ JAMBAQUÉ 1
}

1 Inserm U663, Paris, University Paris Descartes, PRES Sorbonne Paris Cité, Paris, CEA, Gif sur Yvette; 2 Centre de Reference Malformations Cardiaques Congénitales Complexes-M3C, Hôpital Necker Enfants Malades, APHP, University Paris Descartes, Paris, France.

Correspondence to Johanna Calderon at Université Paris Descartes - Centre Henri Piéron UFR Institut de Psychologie, 71 avenue Edouard Vaillant, 92774 Boulogne-Billancourt Cedex, France. E-mail: johanna.calderon@inserm.fr

\begin{tabular}{|c|c|}
\hline \multicolumn{2}{|c|}{ PUBLICATION DATA } \\
\hline $\begin{array}{l}\text { Accepted } \\
2013 .\end{array}$ & for publication 2nd December \\
\hline \multicolumn{2}{|c|}{ Published online 21st January 2014.} \\
\hline \multicolumn{2}{|c|}{ ABBREVIATIONS } \\
\hline $\mathrm{CHD}$ & Congenital heart disease \\
\hline СРB & Cardiopulmonary bypass \\
\hline SES & Socio-economic status \\
\hline TEC & Test of Emotion Comprehension \\
\hline TGA & $\begin{array}{l}\text { Transposition of the great } \\
\text { arteries }\end{array}$ \\
\hline ToM & Theory of mind \\
\hline TREFE & $\begin{array}{l}\text { Test of Facial Recognition of } \\
\text { Emotions for Children }\end{array}$ \\
\hline
\end{tabular}

\section{PUBLICATION DATA}

Accepted for publication 2nd December 2013.

Term infants born with cyanotic congenital heart disease (CHD), including transposition of the great arteries (TGA) have been reported to have significant disturbances in cerebral oxidative metabolism ${ }^{1}$ and mild to moderate white matter injuries primarily in the form of periventricular leukomalacia before and after neonatal open heart surgery. ${ }^{2,3}$ Several medical and patient-related risk factors including pre-, intra-, and postoperative variables may contribute to their neurological status and long-term neurocognitive outcomes. ${ }^{4}$ Despite overall normal general intelligence, ${ }^{5}$ longterm cognitive impairments as well as a high prevalence of attention-deficit-hyperactivity disorder, ${ }^{6}$ 'externalizing' problems (e.g. aggressive behaviour), and 'internalizing' difficulties (i.e. social withdrawal) ${ }^{7}$ have been reported. Recent studies have also demonstrated significant impairments in understanding other people's inner emotional states, suggesting deficits in social cognition. ${ }^{8}$ Compared with children with typical development, children with TGA have been reported to display impairments on mental state attribution (first- and second-order theory of mind
[ToM] tasks) as they had significant difficulties on making correct inferences on the false-beliefs held by others and on understanding and predicting their behaviour. ${ }^{9,10}$ Adolescents with TGA also had significantly worse performances on a test of complex inner state attribution (reading the mind in the eyes test) and exhibit more autistic traits than expected in the general population. ${ }^{11}$ These findings opened a new window for the study of neurocognitive consequences associated with CHD; however, important questions remain concerning the extent and generalization of these difficulties to the broad domain of social cognition.

Social cognition is a multifaceted concept that includes elementary processes such as the recognition of facial expressions of emotion and more complex abilities referring to the comprehension of mental and affective states. ${ }^{12}$ In typical development, early improvements on basic facial emotion recognition are reported in preschool-aged children. ${ }^{13}$ This progress parallels the comprehension of some affective states, such as the understanding that people may 
hold different desires ${ }^{14}$ or that emotions may have external causes (e.g. if somebody gets a present for their birthday, how would they feel?). ${ }^{15}$ Cognitive mental state attribution also undergoes significant progress during this period as children can recognize first-order false-beliefs. ${ }^{16}$ Furthermore, in middle childhood, at around 7 to 8 years, children are able to understand the possibility of hiding emotions (e.g. showing a happy face but feeling sad inside), ${ }^{15}$ and they come to understand second-order falsebeliefs with affective contents (e.g. Paul thinks that Mary feels) followed by epistemic contents (Paul thinks that Mary thinks). ${ }^{17,18}$ Finally, at around 9 to 11 years, they begin to master mixed emotions (e.g. feeling happy and sad at the same time) and some aspects of morality (e.g. feeling guilt after telling lies). ${ }^{15}$ From a neurofunctional point of view, emotion recognition and comprehension in typical development involves networks including the amygdala ${ }^{19}$ and the prefrontal cortex. ${ }^{20}$ Furthermore, concomitant deficits in facial expression recognition and emotion understanding as well as in affective and cognitive ToM have been found in 6- to 8-year-old children after traumatic brain injury ${ }^{21}$ and in children with attentiondeficit-hyperactivity disorder. ${ }^{22}$ Despite the growing concern regarding systematic neurocognitive screening and remediation strategies for children with $\mathrm{CHD},{ }^{23}$ no data concerning the extent and nature of social cognition impairments including emotional processing are available.

The objective of this study was to assess core components of social cognition including elementary aspects such as basic facial expression recognition (perceptual identification and labelling), as well as main milestones of emotion comprehension organized hierarchically according to their developmental path of acquisition (e.g. from desires to moral feelings comprehension). In addition, as school-aged children with TGA have been reported to have significant delays on the litmus test of ToM (cognitive false-belief attribution), ${ }^{9}$ we sought to investigate if delays would also be observed for same-structured tests with an affective component (second-order false-belief tasks with a positive or a negative emotional outcome). In typical development, affective components in second-order false-belief understanding seem to facilitate children's performances. ${ }^{17} \mathrm{We}$ sought to assess if children with TGA would also benefit from this affective effect. Finally, we investigated potential neonatal medical risk factors including pre-, intra-, and postoperative variables associated with emotional and cognitive outcomes.

\section{METHOD}

\section{Participants}

All eligible children born between 2003 and 2005 at the French referral centre for complex congenital heart disease were enrolled in a prospective study of neurocognitive outcomes after neonatal open-heart surgery for D-transposition of the great arteries. No magnetic resonance imaging data were collected. Inclusion criteria included a diagnosis of TGA with or without a ventricular septal defect

\section{What this paper adds}

- Children with TGA do not display significant deficits in facial emotion recognition.

- Children with TGA display significant delays on age-expected measures of emotion comprehension.

- Affective false-belief understanding is also altered for a significant percentage of children with TGA.

- Preoperative variables may play a significant role in the development of sociocognitive abilities after TGA.

(VSD) requiring one arterial switch operation under fullflow cardiopulmonary bypass $(\mathrm{CPB})$ support (mean $\mathrm{CPB}$ duration $132 \mathrm{~min}$; SD 22min) with no requirements for deep hypothermic circulatory arrest (mean age at operation $7 \mathrm{~d})$. Exclusion criteria were birthweight less than $2500 \mathrm{~g}$, the presence of genetic syndromes, complex associated cardiovascular anomalies including aortic arch reconstruction or extracardiac pathologies as well as additional surgical procedures. All exclusion criteria information was extracted from medical records. Among children who met one or more of these criteria, two were excluded because of the presence of a 22q11 deletion. Genetic screening was conducted if clinical concerns were reported. Normal cardiac condition, parent's consent to participation, and geographic location (Paris region) were also taken into account for children's enrolment in the study. Forty-five eligible children were initially enrolled and first examined in 2009 (see Calderon et al. ${ }^{10}$ for further information on this cohort), of which seven were lost to follow-up for the present study. Thirty-eight children with TGA participated in this study (11 females, 27 males; mean age $7 \mathrm{y} 3 \mathrm{mo}$, SD $1 \mathrm{y}$ $2 \mathrm{mo})$. Medical related variables were examined. These included time of diagnosis (prenatal vs postnatal), presence or absence of a ventricular septal defect, birthweight, gestational age, Apgar score at 5 minutes, preoperative metabolic acidosis $(\mathrm{pH}<7.2)$, the need for balloon-atrial septostomy, age at arterial switch operation, total bypass time, total cross-clamp time, and total postoperative intensive care unit stay. No significant differences were found between participants and non-participants for all medical related variables $(p>0.05)$.

Forty-five comparison children were also initially enrolled and first examined at a mean age of 5 years, of whom 14 were lost to follow-up for the present study. Comparison children were recruited from public kindergartens and elementary schools in the same geographic area as children with TGA over the same time period. One or two classes per school were randomly chosen and exclusion criteria were a known developmental or physical impairment, confirmed or suspected intellectual disability, and/or mother language other than French. Parents of all children meeting the inclusion criteria received a letter of information and a consent form. A ratio of one female to three males was applied to match TGA sex ratio by means of simple randomization among females in each classroom. Eighty per cent of eligible families gave consent for participation. Data presented here are from the last evaluation of 
our cohort at a mean age of 7 years. A total of 31 comparison children were re-evaluated (seven females, 24 males; mean age $7 \mathrm{y} 4 \mathrm{mo}$, SD 1y $1 \mathrm{mo}$ ). Parents of all comparison children included in this study provided information on children's health status, medical antecedents, and demographic variables. No abnormal data were reported for any child in the comparison group. Parental educational level and socio-economic status (SES) were recorded for all children. The hospital ethics committee approved this study and parental consent was obtained for participation.

\section{Neuropsychological assessment}

Global Non-verbal IQ was evaluated using the Columbia Mental Maturity Scale ${ }^{24}$ and language was explored with the comprehension subtest from the Neuropsychological Assessment Battery (NEPSY). ${ }^{25}$ Facial expression recognition was assessed with the Test of Facial Recognition of Emotions for Children (TREFE). ${ }^{26,27}$ The Test of Emotion Comprehension (TEC) ${ }^{15,28}$ was used to assess different components of emotion understanding with gradual complexity. Affective false-belief was evaluated with a positive and a negative outcome task. ${ }^{17}$ Cognitive false-belief was evaluated with a standard first- and second-order falsebelief task. $^{29,30}$ All tests are commonly used in clinical and experimental settings with typical and atypical populations and have age-standardized scores based on scores of the general population reported in the literature. The first author (JC), a trained neurodevelopmental psychologist, conducted all assessments. Details are presented in Appendix S1 (online supporting information).

\section{Test of Facial Recognition of Emotions for Children (TREFE)}

The TREFE ${ }^{26,27}$ is composed of 102 faces, 17 per emotional expression (happiness, fear, anger, disgust, sadness, neutrality). The tasks began with six training trials where children received positive or negative feedback depending on their accuracy. No feedback was given during the further trials. Assessment order remained fixed across participants and no time limit was set. First, during the labelling task, each face is presented one by one to the participant, who is asked to label the emotion expressed by the face. Then, a verbal matching task is proposed to control for correct visuo-perceptive processing of facial emotional expressions. The participant is asked to choose the faces that express the three emotions enunciated successively by the experimenter. Six faces (one per emotion, three targets and three foils) are presented at a time. For both tasks, accuracy is evaluated by the number of correct answers per emotion (/8) and a total score of emotion recognition is computed (/48) for the labelling task and the verbal matching task separately.

\section{Test of Emotion Comprehension (TEC)}

The TEC $^{15,28}$ is administered by using a picture book with cartoon scenarios in the upper section and four possible facial emotional expressions among five possible outcomes (sad, angry, scared, happy, and neutral) beneath each scenario. A short story is read about the depicted character (s), whose face was left blank, and children were asked to attribute an emotion to the character by pointing to the correct outcome below. Participants' responses were nonverbal and closed. No feedback was given. The general level of emotion comprehension is measured by the number of correct answers for all components (range 0-9), which are also grouped into three main categories according to their level of complexity. The first category 'external emotions' includes the recognition of facial expressions, understanding of the causes of emotions, and the impact of external events on emotions. The second category 'mental emotions' includes the comprehension of desires, affective first-order false belief and the distinction between outwardly shown and inner emotions. The third and more complex category 'reflective emotions' focuses on the comprehension of concurrent mixed feelings, strategies for emotional regulation, and morality. For each category, scores can range from 0 (all components are failed) to 3 (all components are passed).

\section{False-belief tasks}

One first- and three-second-order false-belief tasks were used. All tasks were mimed using small puppets in a standardized scenario. The two most common versions of the standard false-belief task (first- and second-order) ${ }^{29,30}$ were proposed to evaluate children's ability to attribute cognitive mental states to a character. Two second-order affective false-belief tasks adapted from the standard version were selected in order to assess the children's ability to attribute mental emotional states. ${ }^{17}$ In these tasks, two stories involving a positive and a negative affective outcome for the main character were presented. Children had to infer the character's false belief about a second character's emotions (How does Peter think Mary feels now?). For all tasks, control questions and one test question per story were asked. Responses were non-verbal for the cognitive false-belief tasks where children pointed to one of the two possible locations, and forced-choice verbal for the emotional false-belief tasks where two possible outcomes were given (e.g. He thinks she feels happy or sad?). Test responses were coded in a binary fashion for all tasks (0 for failure, 1 for success). No feedback was given.

\section{Data analyses}

Group (children with TGA vs comparison children) differences were analyzed using Student's $t$-test for independent measures or non-parametric $\chi^{2}$ tests for numerical and categorical variables. Statistical analyses were performed separately on scores on the TREFE labelling and matching tasks, using a mixed analysis of variance (ANOVA) with group as a between-participants factor, and emotion (happiness vs fear vs anger vs disgust vs sadness vs neutral) as a within-participant factor. A one-way ANOVA was computed to elicit the effect of group on the number of categories achieved on the TEC. Analyses of covariance (ANCOVAs) were then used to determine the possible 
confounding effect of language comprehension, age, SES, and sex on the group effects for both TREFE and TEC measures. Post-hoc comparisons were conducted using the Bonferroni test with a $p$ value of $<0.05$ threshold. Proportions of children who succeeded or failed on the falsebelief tasks were compared between groups using non-parametric $\chi^{2}$ tests. Multiple logistic regression analyses for group differences in false-belief tasks controlled for language, age, SES, and sex. McNemar $\chi^{2}$ tests for paired groups were used to compare success rates between affective and non-affective second-order false-belief tasks. Influences of medical variables including all pre-, intra-, and postoperative factors were analyzed using multivariate regression analysis and multiple logistic regression analysis depending on the nature of the score analyzed (numeric for the TREFE and TEC, dichotomic for the ToM tasks). We reported non-standardized effect sizes for mean differences, $95 \%$ confidence intervals (CIs) for all group comparisons and standard errors (SE) for linear regression analyses. Finally, when data were missing, a method of case-wise deletion was used. Statistical analyses were performed with Statistical software (version 9.1; StatSoft Inc., Tulsa, OK, USA).

\section{RESULTS}

\section{Demographic characteristics, IQ, and language scores}

As shown in Table I, children with TGA did not significantly differ from comparisons for SES scores, father's educational level, and mother's educational level. Age was not significantly different between groups and neither was sex. Non-verbal IQ levels were within normal ranges for all children and did not significantly differ between groups. Raw language comprehension scores were significantly lower for children with TGA. When converted to normalized scores reported by the NEPSY for 7-year-olds, however, children with TGA had a mean standard score of 11 , which is considered within the normal range.

\section{Test of facial emotion recognition (TREFE)}

Analyses of variance indicated that children with TGA had a significantly lower total score (mean $[\mathrm{SD}]$ ) on the
TREFE labelling task (41.5 [2.58]) than comparison children (42.8 [2.02]; $F_{1,66}=5.35, p=0.02$, mean difference $-0.27 ; 95 \% \mathrm{CI}-2.4$ to -0.13$)$. However, when confounding variables (age, SES, sex, and language scores) were included in an ANCOVA, this difference between the groups was no longer significant $\left(F_{1,51}=1.80, p=0.18,95 \%\right.$ CI -0.51 to 0.10$)$. Moreover, there was no significant difference between groups on the TREFE matching task for the group with TGA (45.4 [2.75]) and for comparisons (45.9 [1.98]; $F_{1,66}=1.01, p=0.32$, mean difference -0.50 , $95 \%$ CI -1.68 to 0.68$)$.

Results show that accuracy on the recognition of negative emotions such as sadness and disgust was significantly worse for both groups at both parts of the TREFE for the labelling task $\left(F_{5,330}=62.59, p<0.001\right)$ and for the matching task $\left(F_{5,330}=11.02, p<0.001\right.$, Tukey HSD $\left.p<0.01\right)$ indicating that these two negative emotions were generally more difficult to identify for all children.

\section{Test of emotion comprehension (TEC)}

Results indicate that the general level of emotion comprehension as measured by the mean number of components (0-9) correctly achieved is significantly lower in the group of children with TGA (6.7 [1.22]) than in the comparison group (7.4 [0.91]; $F_{1,67}=5.4, \quad p=0.02$, mean difference $-0.61,95 \%$ CI -1.14 to -0.08$)$. ANCOVA controlling for age, language scores, SES, and sex showed a significant effect of language $\left(F_{1,54}=7.79, p=0.008,95 \%\right.$ CI $\left.0.03-0.24\right)$ but no significant effect of age $\left(F_{1,54}=0.80, p=0.37,95 \%\right.$ CI -0.17 to 0.44 ), SES $\left(F_{1,54}=0.11, p=0.73,95 \% \mathrm{CI}-0.33\right.$ to $0.23)$, sex $\left(F_{1,54}=1.05, p=0.30,95 \% \mathrm{CI}-0.15\right.$ to 0.48$)$, and no differences between the groups $\left(F_{1,54}=2.47, p=0.12\right.$, $95 \%$ CI -0.57 to 0.06 ).

Components of the TEC were grouped into three categories of gradual complexity. Results from the ANOVA with post-hoc testing (Bonferroni test) indicated that performances of children with TGA $(2.4$ [0.60]) are significantly lower than comparisons' $(2.9[0.37])$ only for the mental emotions category $\left(F_{3,65}=4.22, p=0.002\right.$, mean difference $-0.39,95 \%$ CI -0.63 to -0.14$)$. This difference persisted in an ANCOVA after controlling for age,

Table I: Demographic characteristics of children with transposition of the great arteries (TGA) and comparison individuals

\begin{tabular}{lcccc} 
Variable & $\begin{array}{l}\text { Group with TGA } \\
(n=38)\end{array}$ & $\begin{array}{c}\text { Comparison } \\
\text { group }(n=31)\end{array}$ & $p^{\text {a }}$ & Mean difference (95\% Cl) \\
\hline Age, y:mo & $7: 3(1: 2)$ & $7: 4(1: 1)$ & 0.72 & $-0.08(-0.56$ to 0.39$)$ \\
Sex, male/female, $n$ & $27 / 11$ & $24 / 7$ & 0.54 & - \\
Father's educational level $^{\mathrm{b}}$ & $3.37(1.56)$ & $3.83(0.97)$ & 0.20 & $-0.46(-1.18$ to 0.25$)$ \\
Mother's educational level $^{\mathrm{b}}$ & $3.38(1.67)$ & $3.16(1.50)$ & 0.58 & $0.21(-0.56$ to 0.99$)$ \\
SES $^{c}$ & $2.15(0.91)$ & $2.09(0.97)$ & 0.78 & $0.06(-0.39$ to 0.51$)$ \\
Non-verbal IQ $_{\text {Language comprehension }^{d}}$ & $113(8.4)$ & $116(9.6)$ & 0.22 & $-2.73(-7.21$ to 1.73$)$ \\
\hline
\end{tabular}

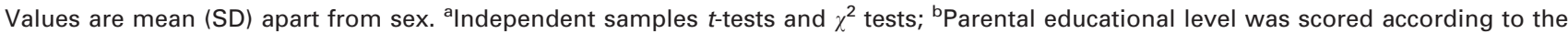
French National Education Diploma classification ( $0=$ primary school education, middle education; $1=$ high school diploma; $2=$ college undergraduate; $3=$ college graduate/masters degree; 4=postgraduate/doctoral degree) ${ }^{\text {c }} \mathrm{SES}$ was scored according to the index from the French National Demographic Statistical Institute. Scores were grouped into four categories: 'very low socio-economic status (SES) $=0$ ', 'Iow $\mathrm{SES}=1$ ', 'intermediate SES $=2$ ', and 'high SES $=3$ '; 'Language was tested using the Neurological Assessment Battery language comprehension subtest. Raw scores are reported here (range 0-28). 
language scores, SES, and sex $\left(F_{1,54}=6.19, p=0.01,95 \% \mathrm{CI}\right.$ -0.34 to -0.03$)$. Results for all three categories are shown in Figure 1.

\section{False-belief tasks}

All children from the comparison group succeeded at the first-order ToM task compared with (30 children) of the children with TGA $\left(\chi^{2}(1)=7.38, p=0.006\right.$; see Fig. 2). There was no significant difference in success rates between the groups for the cognitive second-order falsebelief task. The percentage of children succeeding at the

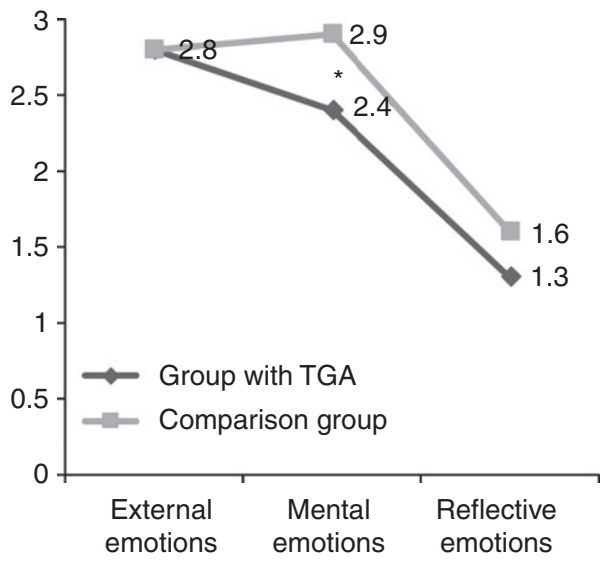

Figure 1: Mean number of components achieved in each category of emotions (external, mental, and reflective) for children with transposition of the great arteries (TGA) versus the comparison group. $* p<0.05$. positive affective second-order false-belief task was significantly lower in the group with TGA $\left(\chi^{2}(1)=4.18, p=0.04\right.$, $95 \%$ CI 0.02 to 1.0$)$. Similarly, in the negative affective second-order false-belief task, the percentage of children with TGA succeeding was significantly lower $\left(\chi^{2}(1)=6.29\right.$, $p=0.01,95 \%$ CI 0.14 to 1.14 ).

Logistic regression analyses also revealed a significant effect of language comprehension score on performances on the first-order task $\left(\chi^{2}(1)=4.13, p=0.04,95 \%\right.$ CI -1.16 to -0.14$)$ and on the negative affective false belief $\left(\chi^{2}(1)\right.$ $=6.59, p=0.01,95 \%$ CI -0.63 to -0.08$)$ tasks. Age, SES, and sex did not have a significant effect on performances on any ToM task. Finally, success rates on the positive and negative affective second-order false-belief tasks were significantly higher compared with the higher order cognitive false-belief task for both groups (respectively $\mathrm{McNemar}$ test $(1)=8.59, p=0.003$ and $M c N e m a r$ test $(1)=9.5$, $p=0.002)$.

\section{Associated medical factors}

Multivariate regression analysis including all medicalrelated variables indicated that the presence of a VSD $\left(\beta=0.48, \mathrm{SE}=0.20, p=0.02, R^{2}=0.32\right)$ and a younger age at the arterial switch operation were significantly associated with better scores at the TREFE labelling task $(\beta=-0.59$, $\left.\mathrm{SE}=0.25, \quad p=0.03, R^{2}=0.56\right)$. Multiple logistic regression analysis showed that prenatal diagnosis of TGA is significantly associated with better performance at the positive emotion false-belief task $\left(\chi^{2}(1)=5.22, p=0.02,95 \%\right.$ CI -3.83 to -0.29$)$. No other significant associations were found between cognitive scores and medical factors.

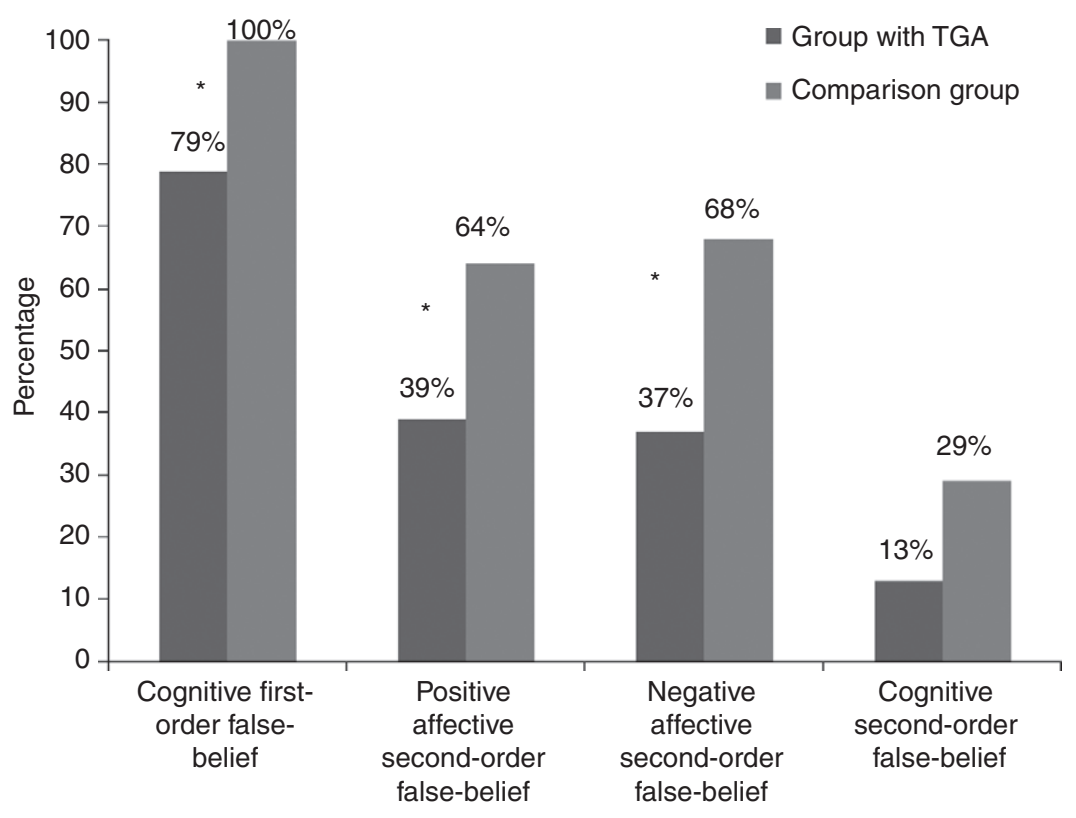

Figure 2: Percentage of children with transposition of the great arteries (TGA) who succedeed at each theory of mind (ToM) task versus the comparison group. $* p<0.05$. 


\section{DISCUSSION}

In recent times there has been a growing concern about social cognition impairments after $\mathrm{CHD},{ }^{23,31}$ yet no research to date has investigated the extent of these deficits. The aim of the current study was to assess the recognition of facial expressions and the gradual comprehension of emotions, as well as the understanding of affective and cognitive false-belief. When compared with the comparison group, children with TGA had significantly lower scores on the test of facial expression labelling despite normal identification of the same emotions when the verbal label was explicitly given. When a series of potential covariates (age, language scores, SES, and sex) was controlled, however, this difference was no longer significant. In typical development, the visuo-perceptive recognition of emotion facial expressions precedes the capacity to produce and correctly retrieve verbal labels for these emotions. ${ }^{13}$ The current results suggest that facial expression recognition generally may be preserved after TGA. Moreover, children with TGA did not exhibit an atypical pattern of performance according to the type of emotion. As observed in typical early development, ${ }^{32}$ negative emotions are less accurately recognized than positive ones and this also applies to our cohort with TGA.

Results demonstrated that children with TGA achieved significantly fewer components in the TEC than comparison children. However, when language comprehension scores, age, SES, and sex were controlled, this group difference disappeared. Indeed, it has been reported that language comprehension levels may explain a significant portion of variance of this test in normal development. ${ }^{33}$ When children's performances were compared according to each category specifically, however, the group with TGA performed significantly less well than the comparison group for the 'mental emotions' category even after controlling for all covariates mentioned including language scores. Children with TGA exhibit significantly lower scores on components such as dealing with desires, affective first-order false-belief or understanding concealed emotions, normally acquired at around 6 to 7 years of age. $^{28}$ According to the gradual complexity of the TEC, our cohort displayed normal scores on the most elementary category taxing external emotions usually achieved at around 5 years of age. ${ }^{28}$ This developmental pattern indicates that basic processing of emotion is not impaired and difficulties apply to age-expected milestones. Thus, their performances were not significantly lower than those observed in the comparison group for the 'reflective emotions' category, which is expected to be acquired later in development. This suggests that children with TGA do not necessarily have an atypical early onset of complex emotion comprehension as their performances were similar to comparison children in the reflective category at age 7 . Instead, it could be hypothesized that developmental lags may become apparent once these abilities have consolidated enough to age-expected levels, even if it is still unclear how these weakness may evolve in the long term. Bellinger et al. ${ }^{11}$ reported that 16-year-olds with TGA are significantly impaired in 'reading the mind in the eyes' test, suggesting that the processing of complex cognitive or affective mental states may have long lasting difficulties. Our data showed that success rates on first-order cognitive false-belief and second-order affective (positive and negative) false-belief were significantly lower for children with TGA and are in accordance with previous findings showing significant impairments in cognitive first- and second-order false-belief understanding in 7-year-old children with TGA. ${ }^{9}$ Taken together, these results add to the evidence for a potential generalization of impairments to emotional and affective components of social cognition. It is worth noting, however, that success rates on affective secondorder false-belief understanding were significantly higher than those on a cognitive, yet similarly structured, secondorder false-belief task. This could mean that the emotional outcome of the task facilitates inner states attribution for children with TGA, comparable to what is observed in typical development. ${ }^{17}$ The success rate of our comparison children at the cognitive second-order task $(29 \%)$ was lower than that reported in a previous study on typical development (in Parker et al. ${ }^{17} 78 \%$ at age 7 ) and lower than our previous cohort of typical 7-year-olds. ${ }^{9}$ This could partially be explained by the inclusion of 6-year-olds in the current study, who systematically failed at this task. It is commonly admitted that typical children can pass second-order false-belief tests between the ages of 7 years and 9 years. ${ }^{30}$ It is also important to note that second-order ToM tasks, especially standard cognitive versions, ${ }^{30}$ have been reported to entail high language comprehension and information processing requirements. Failure at this task could reflect not only difficulties in ToM conceptual understanding per se, but also deficits in decoding and integrating this information as a result of limited linguistic and/or executive abilities. ${ }^{18}$ An important step in dissociating these deficits would be to test children with a linguistically facilitated version of second-order tasks that have been reported to elicit better performances in children with typical development. ${ }^{34}$

Neural correlates of emotion processing including decoding facial emotion expressions and emotional ToM in typical development have been reported to involve cortical and sub-cortical structures including the amygdala, ${ }^{19}$ the orbitofrontal cortex ${ }^{20}$ medial prefrontal cortex, and the right temporo-parietal junction. ${ }^{35}$ These studies have reported an increased neural specialization of these structures $^{35}$ and, more importantly, a crucial role of neural connectivity as white matter volumes progressively increase with age during childhood. ${ }^{36}$ The functional relationships between regions underlying higher-order cognitive skills such as social cognition may require the integrity of association white matter tracts, as has been proposed in adult models. $^{37,38}$ Term infants with cyanotic CHD requiring neonatal interventions have been reported to present white 
matter anomalies and delays in myelination, similar to what can be observed in preterm infants. ${ }^{1,2}$ No study to date has established an association between neonatal white matter disturbances and long-term neurocognitive outcomes in cardiac patients. It is critical to understand the impact that these early anomalies could have on the development of complex cognitive skills known to rely on intact brain connectivity throughout childhood.

Our data showed that among the pre-, intra-, and postoperative variables examined, preoperative factors only (presence of a VSD, younger age at the arterial switch operation, and a prenatal diagnosis of TGA) were significantly associated with better cognitive results on facial expression recognition and affective false-belief. An associated VSD plays a crucial role in cyanosis tolerance in the newborn infant as it allows for mixing of the arterial and pulmonary circulations and, therefore, improvement of oxygen saturation before cardiac surgery. ${ }^{39}$ Younger age at the arterial switch operation has been associated with a reduced risk for white matter injury in the form of periventricular leukomalacia in newborns with TGA and overall preoperative brain injury seems related to severity and duration of exposure to hypoxaemia in these patients. ${ }^{3}$ Finally, our results are in accordance with previous data showing a beneficial impact of prenatal diagnosis on neurocognitive outcomes in preschool-aged children with TGA. ${ }^{10}$ Indeed, prenatal diagnosis of cyanotic CHD is associated with reduced neonatal morbidity including better haemodynamic stability and neurological status as it reduces the odds of prolonged cyanosis before cardiac surgery. ${ }^{39}$

Data from this study may suggest concomitant vulnerabilities in all core aspects of social cognition including ageexpected emotion-processing abilities. Nevertheless, several limitations should be considered. As we sought to limit potential medical confounding variables including multiple cardiac operations and chronic cyanosis, we focused on patients who had TGA as they constitute a homogeneous group with cyanotic CHD and no associated genetic syndromes. Our results cannot be generalized to all children with CHD as variations in haemodynamic instability and operative management should be noted. Our study does not allow for investigation of the neuropathology related to cognitive dysfunction because no brain imaging was available for our patients. In addition, as ToM is also dependent on language development, ${ }^{40}$ future evaluations should systematically test this factor not only as a control variable but also including less demanding ToM tasks in terms of linguistic abilities. Moreover, observational studies (contrary to randomized controlled trials) may be subject to biases, notably participant selection and multiple confounding variables. We provided traditional statistical methods of adjustment for baseline covariates in all our models. Covariance analyses, however, should be conducted with caution and future studies in this population should consider the use of propensity score methods for optimal bias reduction. ${ }^{41}$

Finally, future studies should determine the association of social cognition and emotion processing impairments assessed with neuropsychological testing to more ecological parental reports of children's functioning. This is particularly relevant as children with heart disease may display high levels of behavioural and emotional problems, ${ }^{8,42}$ indicating that daily life social functioning may be vulnerable in these patients. Recently, a scientific statement from the American Heart Association ${ }^{23}$ categorized children requiring open-heart surgery as at high risk for developmental disorders and recommended formal evaluations for these children. Social cognition screening should also be routinely conducted in all patients with CHD at high-risk, as deficits could affect social and emotional outcomes. Remediation or preventive interventions based on formal cognitive training such as 'thought-bubble ToM training' including pictures to depict mental states visually ${ }^{43}$ or interventions based on reinforcement of executive functions, ${ }^{44,45}$ may help reduce social cognition deficits after CHD.

\section{ACKNOWLEDGEMENTS}

We are grateful to all families who participated in this study. This study was supported by the Association pour la Recherche en Cardiologie du Foetus à l'Adulte (ARCFA) and by a university doctoral fellowship to JC.

\section{SUPPORTING INFORMATION}

The following additonal material may be found online:

Appendix S1: Details of assessments used in the study.

\section{REFERENCES}

1. Miller SP, McQuillen PS, Vigneron DB, et al. Preoperative brain injury in newborns with transposition of the great arteries. Ann Thorac Surg 2004; 77: 1698-706.

2. Licht DJ, Shera DM, Clancy RR, et al. Brain maturation is delayed in infants with complex congenital heart defects. 7 Thorac Cardiovasc Surg 2009; 137: 529-36.

3. Petit CJ, Rome JJ, Wernovsky G, et al. Preoperative brain injury in transposition of the great arteries is associated with oxygenation and time to surgery, not balloon atrial septostomy. Circulation 2009; 119: 709-16.
4. Wernovsky G, Shillingford AJ, Gaynor JW. Central nervous system outcomes in children with complex congenital heart disease. Curr Opin Cardiol 2005; 20: 94-9.

5. Bellinger DC, Wypij D, duPlessis AJ, et al. Neurodevelopmental status at eight years in children with dextrotransposition of the great arteries: the Boston Circulatory Arrest Trial. 7 Thorac Cardiovasc Surg 2003; 126: 1385-96.

6. Shillingford AJ, Glanzman MM, Ittenbach RF, Clancy RR, Gaynor JW, Wernovsky G. Inattention, hyperactivity, and school performance in a population of school-age children with complex congenital heart disease. Pediatrics 2008; 121: e759-67.

7. Bellinger DC. Are children with congenital cardiac malformations at increased risk of deficits in social cognition? Cardiol Young 2008; 18: 3-9.

8. Bellinger DC, Newburger JW, Wypij D, Kuban KCK, duPlesssis AJ, Rappaport LA. Behaviour at eight years in children with surgically corrected transposition: the Boston Circulatory Arrest Trial. Cardiol Young 2009; 19: 86-97. 
9. Calderon J, Bonnet D, Courtin C, Concordet S, Plumet $\mathrm{M}-\mathrm{H}$, Angeard N. Executive function and theory of mind in school-aged children after neonatal corrective cardiac surgery for transposition of the great arteries. Dev Med Child Neurol 2010; 52: 1139-44.

10. Calderon J, Angeard N, Moutier S, Plumet M-H, Jambaqué I, Bonnet D. Impact of prenatal diagnosis on neurocognitive outcomes in children with transposition of the great arteries. 7 Pediatr 2012; 161: 94-8.

11. Bellinger DC, Wypij D, Rivkin MJ, et al. Adolescents with d-transposition of the great arteries corrected with the arterial switch procedure: neuropsychological assessment and structural brain imaging. Circulation 2011; 124: $1361-9$

12. Adolphs R. Neural systems for recognizing emotion. Curr Opin Neurobiol 2002; 12: 169-77.

13. Vicari S, Reilly JS, Pasqualetti P, Vizzotto A, Caltagirone C. Recognition of facial expressions of emotions in school-age children: the intersection of perceptual and semantic categories. Acta Paediatr 2000; 89: 836-45.

14. Wellman HM, Liu D. Scaling of theory-of-mind tasks. Child Dev 2004; 75: 523-41.

15. Pons F, Harris PL, de Rosnay M. Emotion comprehension between 3 and 11 years: developmental periods and hierarchical organization. Eur 7 Dev Psychol 2004; 1: $127-52$.

16. Carlson SM, Moses LJ, Claxton LJ. Individual differences in executive functioning and theory of mind: an investigation of inhibitory control and planning ability. 7 Exp Child Psychol 2004; 87: 299-319.

17. Parker JR, MacDonald CA, Miller SA. 'John thinks that Mary feels..' False belief in children across affective and physical domains. 7 Genet Psycbol 2007; 168: 43-61.

18. Miller SA. Children's understanding of second-order mental states. Psychol Bull 2009; 135: 749-73.

19. Somerville LH, Fani N, McClure-Tone EB. Behavioral and neural representation of emotional facial expressions across the lifespan. Dev Neuropsychol 2011; 36: 408-28.

20. Brink TT, Urton K, Held D, et al. The role of orbitofrontal cortex in processing empathy stories in 4- to 8year-old children. Front Psychol 2011; 2: 80.

21. Tonks J, Williams WH, Frampton I, Yates P, Wall SE, Slater A. Reading emotions after childhood brain injury: case series evidence of dissociation between cognitive abilities and emotional expression processing skills. Brain Inj 2008; 22: 325-32.

22. Da Fonseca D, Seguier V, Santos A, Poinso F, Deruelle C. Emotion understanding in children with ADHD Child Psychiatry Hum Dev 2009; 40: 111-21.

23. Marino BS, Lipkin PH, Newburger JW, et al. Neurodevelopmental outcomes in children with congenital heart disease: evaluation and management: a scientific statement from the American Heart Association. Circulation 2012; 126: 1143-72.

24. Burgemeister L, Blum H, Lorge I. Columbia Mental Maturity Scale. New York: Psychological Corporation, 1972.

25. Korkman M, Kirk U, Kemps S. NEPSY: A Developmental Neuropsychological Assessment Manual. San Antonio, TX: Psychological Corporation, 1998.

26. Golouboff N, Fiori N, Delalande O, Fohlen M, Dellatolas G, Jambaqué I. Impaired facial expression recognition in children with temporal lobe epilepsy: impact of early seizure onset on fear recognition. Neuropsychologia 2008; 46: 1415-28.

27. Pinabiaux C, Bulteau C, Fohlen M, et al. Impaired emotional memory recognition after early temporal lobe epilepsy surgery: the fearful face exception? Cortex 2013; 49: 1386-93.

28. Pons F, Harris PL. Longitudinal change and longitudinal stability of individual differences in children's emotion understanding. Cogn Emot 2005; 19: 1158-74.

29. Wimmer H, Perner J. Beliefs about beliefs: representations and constraining function of wrong beliefs in young children's understanding of deception. Cognition 1983; 13: 103-28.

30. Perner J, Wimmer H. 'John thinks that Mary thinks that...': attribution of second-order beliefs by 5 - to 10 year-old children. 7 Exp Child Psychol 1985; 39: 437-71.

31. Bellinger DC. Theory of mind deficits in children with congenital heart disease. Dev Med Child Neurol 2010; 52 1079-80.

32. Gao X, Maurer D. A happy story: developmental changes in children's sensitivity to facial expressions of varying intensities. 7 Exp Child Psychol 2010; 107: 67-86.

33. Pons F, Lawson J, Harris P, de Rosnay M. Individual differences in children's emotions understanding: effects of age and language. San 7 Psychol 2003; 44: 347-53.
34. Coull G, Leekam SR, Bennett M. Simplifying secondorder belief attribution: what facilitates children's performances on measures of conceptual understanding? Soc Dev 2006; 15: 548-63.

35. Saxe RR, Whitfield-Gabrieli S, Scholz J, Pelphrey KA. Brain regions for perceiving and reasoning about other people in school-aged children. Child Dev 2009; 80: 1197-209.

36. Durston S, Hulshoff Pol HE, Casey BJ, Giedd JN, Buitelaar JK, van Engeland H. Anatomical MRI of the developing human brain: what have we learned? $7 \mathrm{Am}$ Acad Child Adolesc Psychiatry 2001; 40: 1012-20.

37. Philippi CL, Mehta S, Grabowski T, Adolphs R, Rudrauf D. Damage to association fiber tracts impairs recognition of the facial expression of emotion. 7 Neurosci 2009; 29: 15089-99.

38. Carrington SJ, Bailey AJ. Are there theory of mind regions in the brain? A review of the neuroimaging literature Hum Brain Mapp 2009; 30: 2313-35.

39. Bonnet D, Coltri A, Butera G, et al. Detection of transposition of the great arteries in fetuses reduces neonatal morbidity and mortality. Circulation 1999; 99: 916-8.

40. Astington JW, Baird J, editors. Why Language Matters for Theory of Mind. Oxford: Oxford University Press, 2005.

41. D'Agostino R. Tutorial in biostatistics, Propensity score methods for bias reduction in the comparison of a treatment to a non-randomized control group. Stat Med 1998; 17: 2265-81.

42. Spijkerboer AW, Utens EMWJ, Bogers AJJC, Verhulst FC, Helbing WA. Long-term behavioural and emotional problems in four cardiac diagnostic groups of children and adolescents after invasive treatment for congenital heart disease. Int 7 Cardiol 2008; 125: 66-73.

43. Paynter J, Peterson C. Further evidence of benefits of thought-bubble training for theory of mind development in children with autism spectrum disorders. Res Autism Spectr Disord 2013; 7: 344-8.

44. Benson J, Sabbagh M, Carlson S, Zelazo P. Individual differences in executive functioning predict preschoolers' improvement from Theory-of-Mind training. Dev Psychol 2013; 49: 1615-27.

45. Diamond A, Barnett W, Thomas J, Munro S. Preschool program improves cognitive control. Science 2007; 318: $1387-8$. 